\title{
COMPOSIÇÃO DE CAROTENÓIDES DE MARACUJÁ-AMARELO (Passiflora edulis flavicarpa) IN NATURA ${ }^{1,2}$
}

\author{
Sandra Regina da SILVA ${ }^{3,4}$, Adriana Z. MERCADANTE ${ }^{3, *}$
}

\section{RESUMO}

A composição de carotenóides de maracujá-amarelo in natura (Passiflora edulis) comercializado em Campinas, Brasil, foi avaliada utilizando-se cromatografia líquida de alta eficiência. Em todas as amostras foram encontrados os seguintes carotenóides: $\beta$-criptoxantina, prolicopeno, cis- $\zeta$-caroteno, $\zeta$-caroteno, $\beta$-caroteno e 13 -cis- $\beta$-caroteno, além de neurosporeno e $\gamma$-caroteno em alguns lotes. Em geral, os teores de carotenóides nos diversos lotes foram significativamente diferentes. Em dois dos cinco lotes analisados, o $\zeta$-caroteno foi o carotenóide principal, contribuindo com 41 e $62 \%$ do total de carotenóides, enquanto que em outros dois lotes, o $\beta$-caroteno foi o majoritário, com 55 e $74 \%$ do total e em um dos lotes o cis- $\zeta$-caroteno esteve presente em maior concentração, com $29 \%$. Este tipo de inversão entre os carotenóides majoritários não é comumente encontrado em frutas e vegetais.

Palavras-chave: carotenóides; maracujá-amarelo; cromatografia líquida de alta eficiência; Passiflora edulis.

\section{SUMMARY}

CAROTENOID COMPOSITION OF FRESH YELLOW PASSION FRUIT (Passiflora edulis). The carotenoid composition of fresh yellow passion fruit (Passiflora edulis) commercialized in Campinas, Brazil, was evaluated by high performance liquid chromatography. In all the samples, the following carotenoids were found: $\beta$-cryptoxanthin, prolycopene, cis- $\zeta$-carotene, $\zeta$-carotene, $\beta$-carotene, 13 -cis- $\beta$-carotene. Besides that neurosporene and $\gamma$-carotene were also found in some lots. In general, the carotenoid contents were significantly different amongst the lots. In two of the five lots, $\zeta$-carotene was the major carotenoid, contributing with 41 and $62 \%$ of the total carotenoid content, while in other two lots $\beta$-carotene was the main carotenoid, with 55 and $74 \%$ of the total carotenoid, and in one of the lots cis- $\zeta$-carotene was present as the major carotenoid, with $29 \%$. The inversion occurring between the main carotenoids is not frequently found in fruits and vegetables.

Keywords: carotenoids; yellow passion fruit; high performance liquid chromatography; Passiflora edulis.

\section{1 - INTRODUÇÃO}

Os carotenóides são corantes naturais de frutas, verduras, raizes, aves, certos peixes, crustáceos e alguns microrganismos. Estes pigmentos de cores que vão do amarelo ao vermelho têm despertado a curiosidade dos cientistas desde o aparecimento da química orgânica devido as suas relevantes funções e ações.

Alguns carotenóides são capazes de serem convertidos em vitamina A e como tal desempenham um importante papel nutricional. Esta função adquire maior importância nos países do terceiro mundo, onde os vegetais e frutos ricos em carotenóides constituem as principais fontes de vitamina A [12]. Os carotenóides também exercem outras ações não relacionadas com a atividade provitaminica A, tais como, diminuição do risco de doenças degenerativas, prevenção da formação de catarata, redução da degeneração macular relacionada ao envelhecimento e redução do risco de doenças coronárias [7]. Além disso, os carotenóides desempenham um papel fundamental como pigmento acessório na fotossintese, agindo como coletor de energia e protetor contra fotooxidação [7].

\footnotetext{
1. Recebido para publicação em 12/03/2001. Aceito para publicação em $07 / 03 / 2002$.

2. Parte da Tese de Doutorado da 1a autora, junto ao Curso de Pós-Graduação em Ciência de Alimentos da FEA/UNICAMP.

3. UNICAMP - Departamento de Ciência de Alimentos - Faculdade de Engenharia de Alimentos, Cx. Postal 6121, CEP 13083-970, CampinasSP.E-mail:mercadan@obelix.unicamp.br.

4. UNOESC - Centro de Ciências Agroambientais e de Alimentos. CX. Postal 187, CEP 89560-000, Videira-SC.

* A quem a correspondência deve ser enviada.
}

Cerca de 150 espécies de Passiflora são nativas do Brasil, das quais mais de 60 produzem frutos que podem ser aproveitados direta ou indiretamente como alimento. O maracujá-amarelo (Passiflora edulis flavicarpa) é o mais cultivado no Brasil e destina-se predominantemente à produção de sucos. A produção brasileira de maracujá supera a de manga, goiaba, e papaya, sendo o Brasil o maior exportador mundial de suco de maracujá [5].

Embora a identidade dos carotenóides de maracujáamarelo in natura já tenha sido confirmada por espectrometria de massas (EM) por MERCADANTE, BRITTON, RODRIGUES-AMAYA [9], a quantificação não foi realizada por estes autores. Já CECCHI \& RODRIGUEZAMAYA [4] quantificaram somente carotenóides de suco comercial de maracujá.

Este estudo teve por objetivo determinar a composição de carotenóides de maracujá-amarelo in natura.

\section{2 - MATERIAL E MÉTODO}

\section{1 - Amostras}

As amostras de maracujá-amarelo ( $P$. edulis flavicarpa) foram adquiridas no comércio de Campinas, Brasil, durante o periodo de abril a julho de 1999. Foram analisados 5 lotes, sendo que cada lote da fruta in natura no estádio completo de amadurecimento consistiu de 4 unidades, cujas polpas foram misturadas e aproximadamente $12 \mathrm{~g}$ foram tomadas para análise. O estádio de amadurecimento foi determinado pela aparência visual da casca completamente amarela, por observação da polpa solta do pericarpo ao se agitar os frutos, e pelos valores de sólidos solúveis (11 a $\left.14^{\circ} \mathrm{Brix}\right)$ e de $\mathrm{pH}(2,7$ a 2,8$)$. 


\section{2 - Análise de carotenóides}

O extrato de carotenóides foi preparado de acordo com MERCADANTE, RODRIGUEZ-AMAYA, BRITTON [10]. As etapas principais foram extração dos pigmentos com acetona e saponificação com $10 \% \mathrm{KOH}$ em metanol por uma noite à temperatura ambiente. Após retirada do álcali, o extrato foi concentrado em evaporador rotatório $\left(\mathrm{T} \leq \mathrm{a} 35^{\circ} \mathrm{C}\right)$ até secar quase completamente e esta etapa foi completada utilizando-se fluxo de $\mathrm{N}_{2}$. A extração foi realizada em duplicata e cada amostra foi injetada duas vezes no cromatógrafo.

Utilizou-se um cromatógrafo líquido de alta eficiência (Waters), equipado com detector de conjunto de diodos (modelo 996), forno para coluna, bomba quaternária (modelo 600), sistema de desgaseificação "on line", injetor "Rheodyne" com alça de amostra de $20 \mu 1$ e sistema de processamento e aquisição de dados Millenium. Os carotenóides foram separados em coluna $\mathrm{C}_{18}$ Nova-Pak $(4 \mu \mathrm{m}$ de tamanho de partícula, 3,9 x 300mm) e acetonitrila/ metanol/acetato de etila $(75: 15: 10)$ como fase móvel, com fluxo de $1 \mathrm{~mL} / \mathrm{min}$, e temperatura da coluna mantida a $29^{\circ} \mathrm{C}$. Os espectros foram adquiridos entre 250 e $600 \mathrm{~nm}$ e os cromatogramas foram processados no comprimento de onda de máximo de absorção $\left(\lambda_{\max }\right)$ de cada carotenóide.

A identificação dos carotenóides foi realizada através de informações obtidas dos espectros de absorção no UVvisivel fornecidos pelo detector de conjunto de diodos, cocromatografia com padrões e ordem de eluição na coluna.

Os padrões de $\beta$-caroteno, $\beta$-criptoxantina e $\zeta$-caroteno foram fornecidos pela F. Hoffmann-La Roche (Basiléia, Suiça). O padrão de prolicopeno foi obtido segundo SILVA \& MERCADANTE [16].

Foram construídas curvas de calibração para os principais carotenóides, com 6 a 9 niveis de concentração, em triplicata. O 13-cis- $\beta$-caroteno e o cis- $\zeta$-caroteno foram quantificados utilizando-se as curvas dos respectivos carotenóides trans. Pontos de concentração conhecida foram injetados diariamente para quantificação das amostras. Os carotenos incolores, fitoeno e fitoflueno, não foram quantificados.

O valor de vitamina A foi calculado de acordo com os fatores de conversão relatados pelo NAS-NRC [11], onde $6 \mu \mathrm{g}$ de $\beta$-caroteno correspondem a $1 \mu \mathrm{g}$ de equivalente de retinol (ER). As bioatividades utilizadas foram: $100 \%$ para all-trans- $\beta$-caroteno, $50 \%$ para all-trans- $\beta$-criptoxantina [2] e de $50 \%$ para o 13 -cis- $\beta$-caroteno.

A precisão entre extrações e injeções foi verificada para os principais carotenóides.

\section{3 - Análise estatística}

A comparação entre as médias, duas a duas, foi feita mediante teste de Tukey, a um nivel de significância de $0,0001[14]$.

\section{3 - RESULTADOS E DISCUSSÃO}

Os valores do coeficiente de variação $(\mathrm{CV})$ obtidos entre extrações variaram de 1,8 a $3,2 \%$ para $\beta$-criptoxantina, de 1,6 a 2,7\% para prolicopeno, de 1,0 a $3,1 \%$ para cis- $\zeta$-caroteno, de 1,9 a $3,6 \%$ para $\zeta$-caroteno e de 1,4 a $3,7 \%$ para $\beta$-caroteno. Estes valores são mais baixos ou comparáveis àqueles relatados para cromatografia líquida de alta eficiência (CLAE) na literatura $[3,6,10,15]$. Injeções em duplicata da mesma extração, como esperado, apresentaram valores de CV mais baixos, como de 0,0 a $1,0 \%$ para $\beta$-criptoxantina, de 0,0 a $1,8 \%$ para prolicopeno, de 0,0 a $0,4 \%$ para cis- $\zeta$-caroteno, de 0,1 a 0,9\% para $\zeta$-caroteno e de 0,0 a 0,9\% para $\beta$-caroteno.

A Figura 1 mostra o cromatograma do extrato de carotenóides do lote $\mathbf{1}$ cujo perfil foi semelhante ao do lote 2. Uma vez que os perfis de carotenóides dos lotes $\mathbf{3}$ e $\mathbf{5}$ foram semelhantes, a Figura 2 apresenta o cromatograma relativo ao lote $\mathbf{3}$. O cromatograma referente ao lote 4 que mostrou um perfil diferente dos demais lotes está na Figura 3.

A identidade dos picos presentes nos cromatogramas e suas características obtidas por CLAE estão apresentadas na Tabela 1. Em todos os lotes foram encontrados os seguintes carotenóides: $\beta$-criptoxantina, prolicopeno, cis- $\zeta$-caroteno, $\zeta$-caroteno, $\beta$-caroteno e 13 -cis- $\beta$-caroteno. $\mathrm{O} \gamma$-caroteno ocorreu em traços somente no lote $\mathbf{3}$ e traços de neurosporeno foi encontrado nos lotes $\mathbf{1}$ e $\mathbf{2}$. O fitoflueno foi encontrado em todos os lotes, porém coeluiu com o $\beta$-caroteno.

Nota-se que o pico 4, presente em todas as amostras, caracterizou-se por ser uma mistura de carotenóides. Os carotenóides contidos nesta mistura co-eluíram em todas as fases móveis testadas para CLAE. Além disso, tentativas de separar esta mistura por cromatografia em coluna aberta (CCA) e cromatografia em camada delgada (CCD), para posterior identificação dos carotenóides, não foram bem sucedidas.

MERCADANTE, BRITTON, RODRIGUES-AMAYA [9], utilizando EM, confirmaram a identidade dos carotenóides encontrados no presente estudo, além de licopeno, anteraxantina, violaxantina, neoxantina e $\beta$-citraurina, este último encontrado somente em um dos três lotes analisados de maracujá in natura. Porém, estes autores não encontraram cis- $\zeta$-caroteno e 13 -cis- $\beta$-caroteno, os quais foram detectados no presente trabalho. Isto se deve ao fato do isolamento dos carotenóides ter sido realizado no trabalho citado por CCA e CCD, ambas de fase normal, que não possibilitam a separação dos isômeros cistrans; além disto a EM é uma técnica que não permite a identificação de isômeros geométricos.

CECCHI \& RODRIGUEZ-AMAYA [4] encontraram em suco comercial pasteurizado de maracujá: $\beta$-caroteno, $\zeta$-caroteno, cis-neurosporeno e neurosporeno. Estes autores [4] reportaram também a presença de traços de acaroteno, $\gamma$-caroteno, licopeno, aurocromo, criptocromo e auroxantina.

Foram observadas concentrações significativamente diferentes $(\mathrm{p}<0,0001)$ entre os mesmos carotenóides nos cinco lotes de frutos de maracujá in natura analisados no presente estudo, como pode ser observado na Tabela 2. 
O $\zeta$-caroteno foi o carotenóide majoritário nos lotes 1 e $\mathbf{2}$, com 34 e $51 \%$ do total de carotenóides, respectivamente. No entanto, nos lotes $\mathbf{3}$ e $\mathbf{5}$, o $\beta$-caroteno foi o principal carotenóide, perfazendo, respectivamente, 70 e $49 \%$ do total. Já no lote 4, o cis- $\zeta$-caroteno apresentou-se em maior proporção (29\%) que o $\beta$-caroteno (27\%) e o $\zeta$-caroteno (16\%).

Verificou-se que houve, também, variação na proporção dos demais carotenóides, e que esta variação foi dependente do carotenóide majoritário presente nas amostras (Tabela 2). Pode-se observar que nos lotes $\mathbf{1}$ e $\mathbf{2}$ que o $\zeta$-caroteno foi o carotenóide principal, o neurosporeno foi detectado em quantidades muito pequenas e as concentrações de prolicopeno foram maiores comparativamente às encontradas nos lotes $\mathbf{3}$ e $\mathbf{5}$ onde o $\beta$-caroteno foi majoritário. Similarmente, nos lotes $\mathbf{3}$ e $\mathbf{5}$ onde o $\beta$-caroteno predominou, a $\beta$-criptoxantina foi encontrada em concentrações maiores que nos lotes onde $\zeta$-caroteno era majoritário e o neurosporeno não foi detectado. Como essas transformações são controladas por enzimas específicas para cada reação, a ação das enzimas que promove a ciclização e posterior hidroxilação dos carotenóides acíclicos foi favorecida nos lotes $\mathbf{3}$ e $\mathbf{5}$, o que resultou na alta concentração de $\beta$-caroteno e de $\beta$-criptoxantina em detrimento das concentrações de $\zeta$-caroteno, de prolicopeno e de neurosporeno.

Algumas variações nas proporções de carotenóides de frutos e vegetais também foram observadas em outros trabalhos. $\beta$-Citraurina foi encontrada em somente um dos três lotes analisados em maracujá in natura [9]. Em abóbora e moranga comercializadas no Estado de São Paulo, Brasil, foram encontradas além de marcantes diferenças qualitativas entre variedades distintas, variações de até oito vezes no teor do carotenóide majoritário nos cinco lotes de amostra de uma mesma variedade [1].

Este tipo de variação também foi observado em alguns lotes de produtos comerciais de maracujá analisados por SILVA \& MERCADANTE [16]. Em uma das quatro marcas de suco pasteurizado, e em uma das três marcas de polpa congelada, o $\zeta$-caroteno foi o carotenóide principal em três lotes, enquanto o $\beta$-caroteno foi o majoritário em dois lotes, em ambos os produtos. Nas outras marcas de suco e polpa, bem como nos outros produtos, o $\zeta$-caroteno esteve sempre presente em maior concentração.

Por outro lado, este fato não foi anteriormente observado em outros trabalhos com maracujá in natura da variedade púrpura [8,13] e suco processado [4]. Entretanto, nestes estudos utilizou-se um número menor de amostras.

Como o $\beta$-caroteno foi o principal carotenóide que contribuiu para o valor de vitamina A e este apresentou variações marcantes em seu conteúdo, valores de vitamina A de 43,63 a 244,50 ER/100g também foram significativamente diferentes entre os lotes analisados, com um nivel de significância de 0,0001 (Tabela 2). Esta variação muito grande pode provavelmente ser explicada pela diferença entre a procedência das amostras (solo e clima) e de grau de maturação pois como o maracujá é uma fruta climatérica apresenta alta taxa respiratória ocasionando mudanças bioquímicas muito rápidas.
TABELA 1. Propriedades, obtidas por ClAE, dos carotenóides de maracujá in natura.

\begin{tabular}{|c|c|c|c|c|}
\hline pico $^{a}$ & carotenóide & $t_{R}(\min )^{b}$ & $\lambda_{\max }(\mathrm{nm})^{\mathrm{c}}$ & \% IIIIIII \\
\hline 1 & $\beta$-criptoxantina & $10,3-11,1$ & $(428), 454,482$ & 29 \\
\hline 2 & prolicopeno & $17,3-18,7$ & (418), 441, (466) & 0 \\
\hline 3 & neurosporeno & $18,9-19,3$ & $417,441,470$ & 96 \\
\hline 4 & mistura & $20,0-21,9$ & $\ldots$ & _- \\
\hline 5 & $\gamma$-caroteno & 20,7 & $(435), 461,490$ & 45 \\
\hline 6 & cis-ל-caroteno & $22,4-24,4$ & $296,378,400,425$ & 89 \\
\hline 7 & $\zeta$-caroteno & $23,5-25,7$ & $381,401,426$ & 104 \\
\hline \multirow[t]{2}{*}{8} & $\beta$-caroteno + & $27,7-30,1$ & $(424), 454,480$ & 25 \\
\hline & fitoflueno & & $333,349,366$ & 103 \\
\hline 9 & 13-cis- $\beta$-caroteno & $29,2-30,7$ & $339,(426), 447,470$ & 12 \\
\hline 10 & fitoeno & $33,6-36,7$ & (273), 285, (293) & 0 \\
\hline
\end{tabular}

a Numerado de acordo com os cromatogramas apresentados nas Figuras 1, 2 e 3. - Faixa de 20 corridas.
c Solvente: acetonitrila/metanol/acetato de etila $(75: 15: 10)$. Parêntesis indica ombro ao invés de pico.

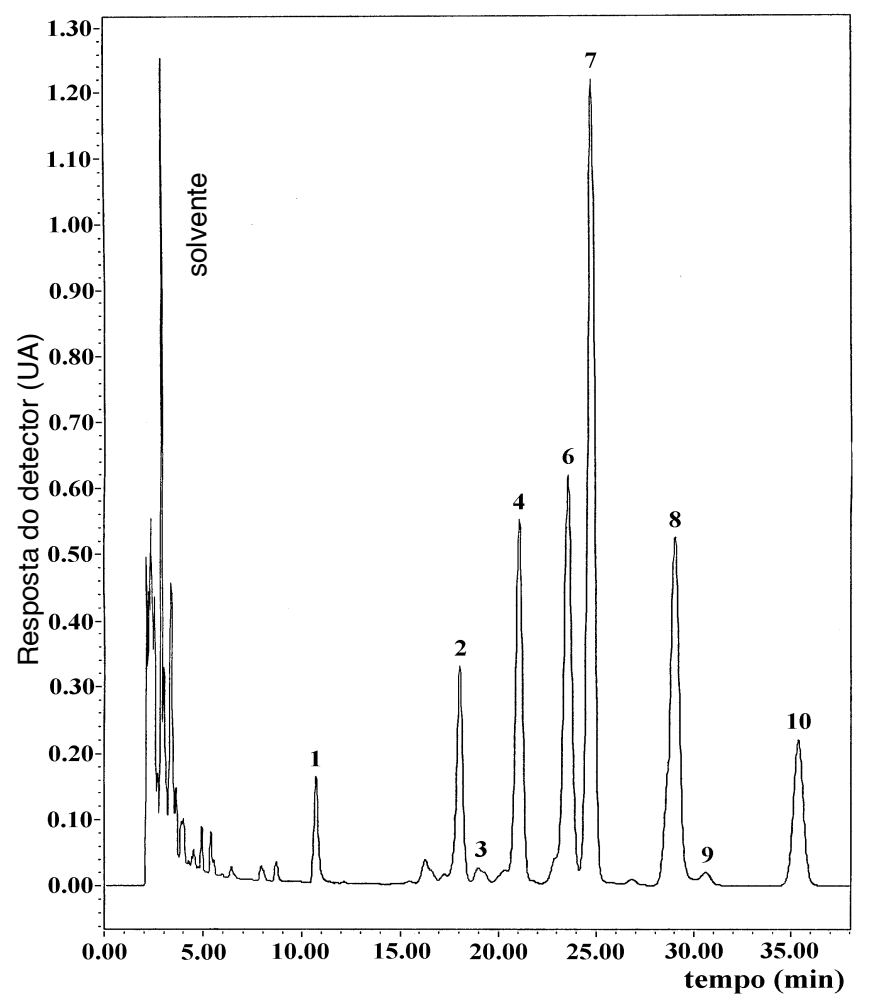

FIGURA 1. Cromatograma, obtido por CLAE, de carotenóides de maracujá do lote 1. Condições cromatrográficas: coluna $\mathrm{C}_{18}$ Nova-Pak $(4 \mu \mathrm{m}, 3,9 \times 300 \mathrm{~mm})$, fase móvel: acetonitrila/ metanol/acetato de etila (75:15:10), fluxo de $1 \mathrm{~mL} / \mathrm{min}$ e temperatura da coluna de $29^{\circ} \mathrm{C}$. Detecção no $\lambda_{\max }$. A identidade dos picos encontra-se na Tabela 1. 


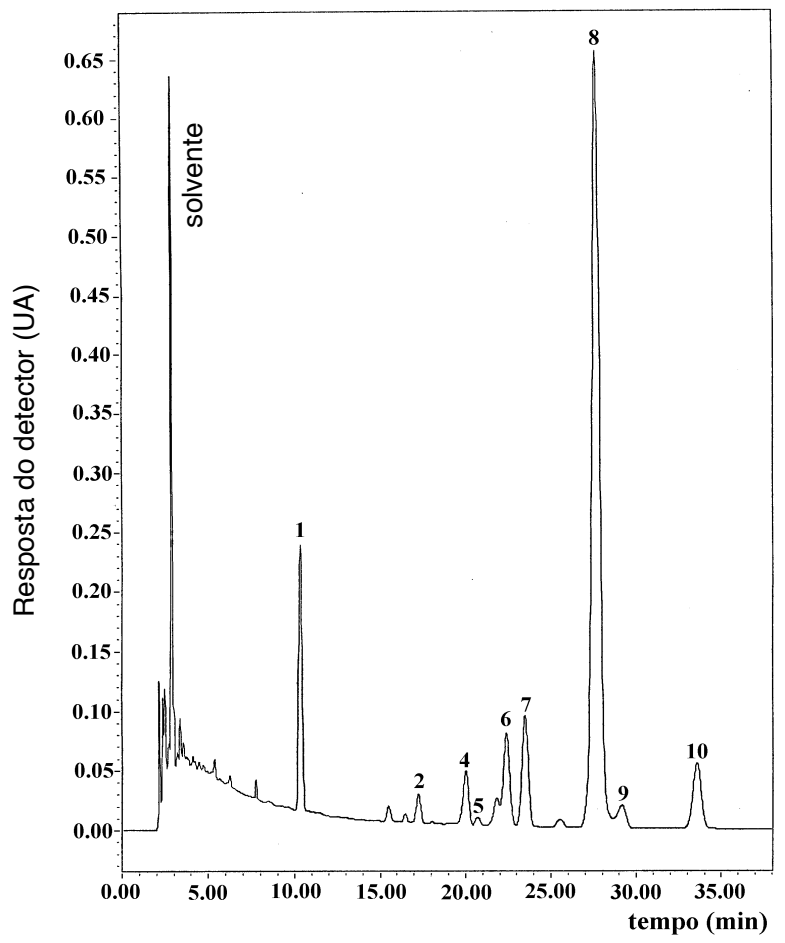

FIGURA 2. Cromatograma, obtido por CLAE, de carotenóides de maracujá do lote 3. Condições cromatrográficas: coluna $\mathrm{C}_{18}$ Nova-Pak (4 $\left.\mu \mathrm{m}, 3,9 \times 300 \mathrm{~mm}\right)$, fase móvel: acetonitrila/ metanol/acetato de etila (75:15:10), fluxo de $1 \mathrm{~mL} / \mathrm{min}$ e temperatura da coluna de $29^{\circ} \mathrm{C}$. Detecção no $\lambda_{\max }$. A identidade dos picos encontra-se na Tabela 1.

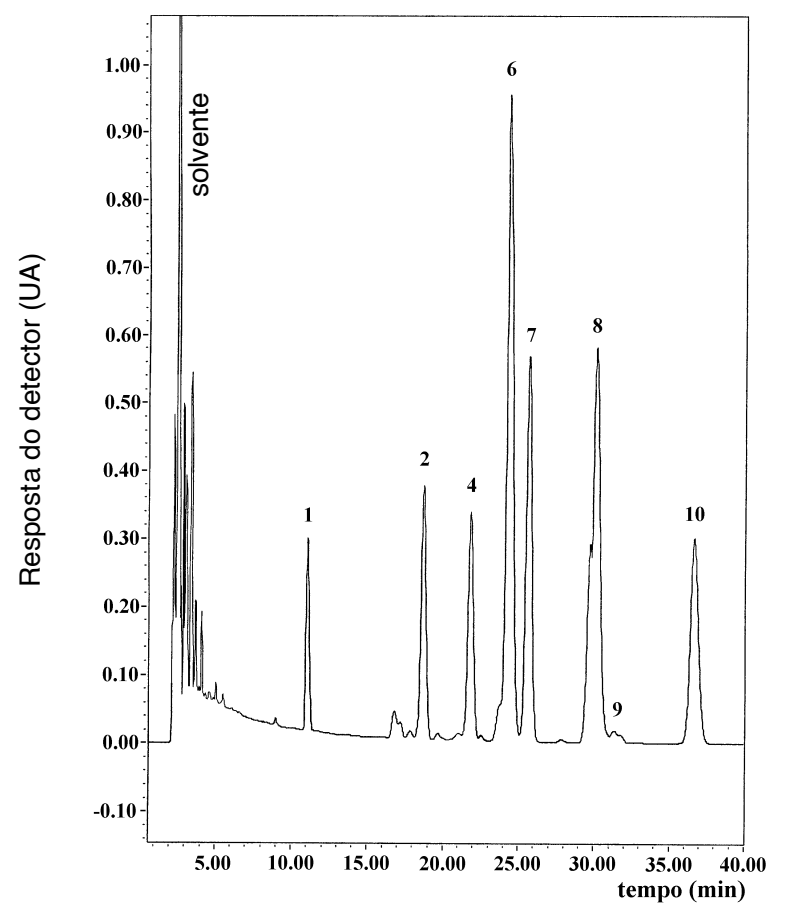

FIGURA 3. Cromatograma, obtido por CLAE, de carotenóides de maracujá do lote 4. Condições cromatrográficas: coluna $\mathrm{C}_{18}$ Nova-Pak ( $\left.4 \mu \mathrm{m}, 3,9 \times 300 \mathrm{~mm}\right)$, fase móvel: acetonitrila/ metanol/acetato de etila (75:15:10), fluxo de $1 \mathrm{~mL} / \mathrm{min}$ e temperatura da coluna de $29^{\circ} \mathrm{C}$. Detecção no $\lambda_{\max }$. A identidade dos picos encontra-se na Tabela 1.
O teor total de carotenóides encontrado em frutos de maracujá-amarelo no presente trabalho (15,36 a $27,14 \mathrm{mg} / \mathrm{g}$ ) foi superior ao encontrado em 2 marcas co-

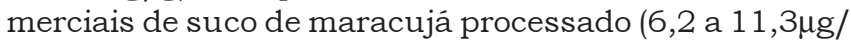
g) [4]. Porém, os valores do presente estudo se encontram muito próximos dos teores relatados por SILVA \& MERCADANTE [16] para 4 marcas de suco de maracujá $(7,16$ a $28,44 \mu \mathrm{g} / \mathrm{g})$ e para 3 marcas de polpa congelada de maracujá $(8,71$ a $23,83 \mu \mathrm{g} / \mathrm{g})$.

TABELA 2. Composição de carotenóides e valor de vitamina A de maracujá in natura.

\begin{tabular}{|c|c|c|c|c|c|}
\hline \multirow{3}{*}{ carotenóide $^{a}$} & \multicolumn{5}{|c|}{ concentração $(\mu g / g)^{b}$} \\
\hline & Iote 1 & Iote 2 & Iote 3 & lote 4 & lote 5 \\
\hline & média $\pm D P$ & média $\pm \mathrm{DP}$ & média $\pm \mathrm{DP}$ & média $\pm \mathrm{DP}$ & média $\pm \mathrm{DP}$ \\
\hline$\beta$-criptoxantina & $0,69 \pm 0,02 \mathrm{a}$ & $0,45 \pm 0,01 \mathrm{~b}$ & $1,27 \pm 0,04 \mathrm{c}$ & $1,75 \pm 0,03 \mathrm{~d}$ & $2,65 \pm 0,09 \mathrm{e}$ \\
\hline prolicopeno & $1,71 \pm 0,03 \mathrm{a}$ & $2,25 \pm 0,06 \mathrm{~b}$ & $0,30 \pm 0,00 \mathrm{c}$ & $3,02 \pm 0,07 d$ & $2,59 \pm 0,04 \mathrm{e}$ \\
\hline neurosporeno & $\operatorname{tr}$ & $\operatorname{tr}$ & ND & ND & ND \\
\hline mistura & $3,69 \pm 0,03 \mathrm{a}$ & $4,59 \pm 0,02 b$ & $0,63 \pm 0,02 c$ & $3,34 \pm 0,04 \mathrm{~d}$ & $2,91 \pm 0,08 \mathrm{e}$ \\
\hline$\gamma$-caroteno & ND & ND & tr & ND & ND \\
\hline 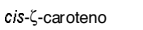 & $4,45 \pm 0,07 \mathrm{a}$ & $2,82 \pm 0,06 \mathrm{~b}$ & $0,74 \pm 0,02 \mathrm{c}$ & $7,38 \pm 0,07 d$ & $2,59 \pm 0,04 \mathrm{e}$ \\
\hline$\zeta$-caroteno & $7,78 \pm 0,21 \mathrm{a}$ & $12,86 \pm 0,46 b$ & $1,26 \pm 0,04 \mathrm{c}$ & $3,93 \pm 0,08 d$ & $3,05 \pm 0,08 \mathrm{e}$ \\
\hline$\beta$-caroteno & $4,48 \pm 0,07 a$ & $2,39 \pm 0,03 \mathrm{~b}$ & $10,79 \pm 0,40 \mathrm{c}$ & $6,77 \pm 0,12 \mathrm{~d}$ & $13,35 \pm 0,31 \mathrm{e}$ \\
\hline 13-cis $\beta$-caroteno & tra & tr a & $0,36 \pm 0,01 b$ & $\operatorname{tra}$ & $\operatorname{tra}$ \\
\hline total & $22,80 \pm 0,37 a$ & $25,37 \pm 0,35 b$ & $15,36 \pm 0,37 c$ & $25,19 \pm 0,20 d$ & $27,14 \pm 0,47 \mathrm{e}$ \\
\hline $\begin{array}{l}\text { vitamina } A \\
(E R / 100 g)\end{array}$ & $80,46 \pm 1,36 a$ & $43,63 \pm 0,53 b$ & $193,46 \pm 6,89 \mathrm{c}$ & $127,42 \pm 1,65 \mathrm{~d}$ & $244,50 \pm 5,77 \mathrm{e}$ \\
\hline
\end{tabular}

a Ordem de eluição na coluna $\mathrm{C}_{18}$ Nova-Pak.

b Média e desvio padrão de duplicatas injetadas duas vezes.

ND: não detectado.

tr: traços (menor que $0,01 \mu \mathrm{g} / \mathrm{g}$.

Valores na mesma linha com letras diferentes foram significativamente diferentes $(\mathrm{p}<0,0001)$.

\section{4 - CONCLUSÕES}

Em todos os lotes de maracujá-amarelo in natura foram encontrados os seguintes carotenóides: $\beta$-criptoxantina, prolicopeno, cis- $\zeta$-caroteno, $\zeta$-caroteno, $\beta$-caroteno e 13 cis- $\beta$-caroteno.

Houve uma grande variação quantitativa nos teores dos diferentes carotenóides nos cinco lotes analisados. $\mathrm{O} \zeta$-caroteno foi encontrado em concentrações de até $62 \%$ do total de carotenóides, sendo o carotenóide principal em 2 dos 5 lotes analisados. O $\beta$-caroteno foi encontrado em concentrações de até $74 \%$ do total, sendo o carotenóide majoritário em outros dois lotes. O cis- $\zeta$-caroteno esteve em maior concentração em 1 dos lotes, com 29\%. O $\beta$-caroteno foi o carotenóide responsável pela maior contribuição para o valor de vitamina A.

\section{5 - REFERÊNCIAS BIBLIOGRÁFICAS}

[1] ARIMA, H.K.; RODRIGUEZ-AMAYA, D.B. Carotenoid composition and vitamin A value of commercial brazilian squashes and pumpkins. Journal of Micronutrient Analysis, v. 4, p. 177-191, 1988.

[2] BAUERFEIND, J.C. Carotenoid vitamin A precursors and analogs in foods and feeds. Journal of Agricultural and Food Chemistry, v. 20, p. 456-473, 1972.

[3] BUSHWAY, R. J. \& WILSON, A. M. Determination of a-and bcarotene in fruit and vegetables by high performance liquid chromatography. Canadian Institute of Food Science and Technology Journal, v. 15, p. 165-169, 1982.

[4] CECCHI, H.M.; RODRIGUEZ-AMAYA, D. Carotenóides e valor de vitamina A em suco de maracujá processado. Ciência e Cultura, v. 33, p. 72-76, 1981. 
[5] CHAN, H.T. Passion fruit, papaya and guava juices. In: NAGY, S.; CHEN, C.S.; SHAW, P.E. (Eds.) Fruit Juice Processing Technology. Agscience Inc.: Auburndale (Flórida), p. 334-348, 1993.

[6] HAMANO, P.S.; MERCADANTE, A.Z. Composition of carotenoids from commercial products of caja (Spondias lutea). Journal of Food Composition and Analysis, v. 13, 2001 (no prelo).

[7] KRINSKY, N.I. The biological properties of carotenoids. Pure \& Applied Chemistry, v. 66, p. 1003-1010, 1994.

[8] LEUENBERGER, F.J.; THOMMEN, H. Zum Vorkommen von carotinoiden in der Passionsfrucht. Zeitschrift fur Lebensmittel-Untersuchung Und-Forschung, v. 149, p. 279-282, 1972.

[9] MERCADANTE, A.Z.; BRITTON, G; RODRIGUEZ-AMAYA, D.B. Carotenoids from yellow passion fruit (Passiflora edulis). Journal of Agricultural and Food Chemistry, v. 46, p. 4102-4106, 1998.

[10] MERCADANTE, A.Z; RODRIGUEZ-AMAYA, D.B.; BRITTON, G. HPLC and mass spectrometric analysis of carotenoids from mango. Journal of Agricultural and Food Chemistry, v. 45, p. 120-123, 1997.
[11] NAS-NRC. Recommended Dietary Allowances, National Academy of Science, Washington. p. 78-92, 1989.

[12] OLSON, J.A. Biological actions of carotenoids. Journal of Nutrition, v. 119, p. 94-95, 1989.

[13] PRUTHI, J.S.; LAL, G. Carotenoids in passion fruit juice. Food Research, v. 23, p. 505-510, 1958.

[14] SAS Institute Inc. SAS/STAT Guide for Personal Computers, version 6.03; SAS Institute: Cary, NC, 1987.

[15] SCOTT, K. J., FINGLAS, P. M., SEALE, R., HART, D. J. and FROIDMONT-GÖRTZ, I. Interlaboratory studies of HPLC procedures for the analysis of carotenoids in foods. Food Chemistry, v. 57, p. 85-90, 1996.

[16] SILVA, S.R.; MERCADANTE, A.Z. Composition of carotenoids from commercial products of passion fruit (Passiflora edulis). Journal of Food Composition and Analysis, 2001 (em preparação).

\section{6 - AGRADECIMENTOS}

Os autores agradecem o auxílio financeiro do CNPq e MCT/FINEP/PRONEX. 